\title{
Multiple scales solution for a beam with a small bending stiffness
}

\begin{abstract}
V. Denoël ${ }^{1}$, E. Detournay ${ }^{2}$
Abstract

This paper considers the problem of a beam with a small bending stiffness, within the framework of a non-linear beam model that includes both the classical cable and the linear beam as limiting cases. This problem, treated as a perturbation of the catenary solution, is solved with the multiple scales method. The resulting expressions of the beam deflection and of the internal forces, as well as those obtained with the more commonly applied matched asymptotics method, are compared with numerical results. This comparison indicates that a better accuracy can be achieved with the multiple scales approach, for a similar computational effort. These results also suggest that application of the multiple scales method to the solution of beam problems involving boundary layers extend the range of values of the small parameter, for which accurate analytical solutions can be obtained by a perturbation technique.
\end{abstract}

Matched asymptotics, multiple scales, boundary layer, catenary, rod theory, small bending stiffness.

\section{Introduction}

Beams are structural elements that resist transverse loads by means of their bending stiffness, while cables (or more traditionally, catenaries) resist transverse loads by means of large displacements and axial forces. There is a wide category of problems, however, for which structural elements exhibit

\footnotetext{
${ }^{1}$ National Fund for Scientific Research, Belgium

University of Liège, Department of Architecture, Geology, Environment and Constructions, Chemin des Chevreuils, 1, Bât B 52/3, 4000 Liège, Belgium

${ }^{2}$ University of Minnesota, Minneapolis, Department of Civil Engineering, 500 Pillsbury Drive S.E. Minneapolis, MN, USA

Commonwealth Scientific and Industrial Research Organisation, Petroleum Resources, 26 Dick Perry Avenue, Kensington, WA 6151, Australia
} 
a behavior intermediate to these two limiting cases. Indeed, cables with a non-negligible bending stiffness or highly taut beams are encountered in many engineering applications, such as clamped stay-cables, suspension cables, electric transmission lines, drillstrings, pipeline installation, and tautstrip models for wind-tunnel measurements. In these structures, boundary layers develop in zones where the inclination is perturbed from the catenary solution, essentially because of locally applied forces or moments. In these zones numerical solutions require careful attention, as small steps or fine meshes are needed to achieve reasonable accuracy. Alternatively, approximate analytical solutions can be constructed by viewing the problem as a singular perturbation of the classical cable problem, with the bending stiffness considered as a small parameter.

Perturbation methods can be classified into two main techniques: matched asymptotics expansion and multiple scales (Hinch, 1991). With the matched asymptotics method, the solution of a perturbed equation is expressed in different scales, each essentially dedicated to a part of the domain. This method is typically applied to solving differential equations characterized by the existence of boundary layers. Indeed, a coordinate at the scale of the whole domain is appropriate for representing the global solution, whereas a stretched coordinate is required to capture the solution inside a boundary layer. On the other hand, the multiple scales method postulates the existence of different scales, without requiring that these scales occur in separate regions of the domain. This method is naturally more general than the matched asymptotics and can also be applied to the solution of boundary value problems, whenever analytical developments remain feasible. The multiple scales method gives a similar but more accurate result than the matched asymptotic expansion for moderate values of the small parameter (Kevorkian and Cole, 1996). This is evidently justified by a better mixing of the different scales.

A review of the literature reveals that the problem of a cable with a small bending stiffness has so far been studied only within the framework of the matched asymptotics method. Early contributions were made by Esipova (1975) and Vasileva and Butuzov (1973), who developed a general theory for boundary value problems that are modeled by singularly perturbed systems of ordinary differential equations. This theory was first applied by Schmeiser (1985) and Schmeiser and Weiss (1986) to the analysis of a beam with hinged and clamped supports. In Schmeiser's pure mathematical developments, the beam is modeled by a set of four differential equations, not all involving the small 
parameter. Wolfe (1993) gave a more formal description of the problem by means of a Cosserat rod (accounting therefore for the extensibility of the cable), and solved a similar set of perturbed differential equations. The matched asymptotic expansion method has been applied to various engineering problems, like conducting wires in magnetic fields (Wolfe, 1991), stay-cables (Irvine, 1993), transport of thin-sheet materials as paper mills, steel sheets, textile fabric (Stump and Fraser, 2000), bending and twisting of rods (Stump and van der Heijden, 2000) and unwinding process from cylindrical yarn packages (Clark et al., 2001).

While addressing applications that deal with drillstrings and hanging cables, Plunkett (1967) appears to be the first to point out the existence of boundary layers and to attempt to construct a matched asymptotics solution. His small rotation hypothesis in the boundary layer led, however, to an erroneous matching, as pointed out by Stump and Fraser (2000). Historically, the first rigorous mathematical development of a non-linear beam with small bending stiffness was given by Flaherty and O'Malley (1982). Based on their seminal work, Rienstra (1987) considered, with a simple beam model, the problem of abandoning and recovering a pipe from a lay barge at sea. The simplicity of the description of the beam used by Rienstra makes his contribution intuitively appealing. His approach serves as a basis for the problem considered in this paper, which is solved using the alternative multiple scales method, however.

In parallel to these theoretical developments, significant efforts have been devoted to the numerical solution of singularly perturbed boundary value problems. Elegant finite difference methods (Jain et al., 1984), finite element methods (Stynes and Oriordan, 1986), shooting methods (Maier, 1986), collocation methods (Ascher and Weiss, 1983; Rao and Kumar, 2007) and mixed methods (Bieniasz, 2008) have been proposed and are still being refined (Kumar et al., 2007). These efforts aim at establishing robust techniques to bypass the meshing problems or the necessity of using small steps in the iterative solution procedure. In spite of considerable research efforts invested in the improvement of algorithms, numerical solutions still require a significant amount of computation time.

All the above referred papers that solve the problem of a cable with a small bending stiffness by perturbation are based on matched asymptotic expansion. The originality of this paper is the construction of a solution for this problem using the multiple scales method. The technique is presented along a fundamental problem, encountered in many engineering applications (Fig.1-a). In particular, 
this problem is part of the solution proposed by Denoël (2008), to analyze the insertion of a beam into a conduit. A beam inserted into a curved conduit adopts its deformed configuration through contacts with the walls. The proposed fundamental problem could thus be seen as the study of the segments of the beam between the contacts. For a small bending stiffness, the semi-analytical method proposed by Denoël (2008) fails to converge, and an analytical solution such as one resulting from an application of the multiple scales method can redress the situation. Published contributions using the same beam model consider a slightly different problem (Fig. 1-b), as the location of the beam ends are not imposed. It will be seen that this problem can be obtained as a particular case of our fundamental problem. Hence the generalization to a wider class of application, studied with one differential equation only, may therefore be seen as a second novelty of this paper.

\section{Fundamental Beam Problem}

The considered fundamental problem involves a beam of length $\ell$, weight per unit length $w$ and constant bending stiffness $E I$, see Fig. 1-a. Given the relative positions $\Delta_{x}$ and $\Delta_{y}$ and the inclinations $\theta_{p}, \theta_{q}$ of the beam ends, we seek to determine the deformed configuration of the beam, as well as the axial and transverse force and moment distribution, under conditions of "small" bending stiffness.

Under the Euler-Bernoulli assumptions, the equation governing the deflection of a beam is (Timoshenko and Goodier, 1987)

$$
E I \frac{d \theta}{d s}=M
$$

where $M(s)$ is the bending moment $\operatorname{and} \theta(s)$ is the beam inclination with respect to the vertical. In the following developments, the exact expression of the curvature is used, namely the derivative of the beam inclination with respect to the curvilinear coordinate $s$. This provides the most general geometrically non-linear beam model, as it accounts for large displacements and large rotations. In the fundamental problem, the end reactions are a priori unknown.

The local equilibrium of the beam in terms of the curvilinear coordinate $s$ (Fig. 2-a) is expressed as

$$
F_{1} \theta^{\prime}+F_{2}^{\prime}-w \sin \theta=0
$$




$$
\begin{aligned}
F_{2} \theta^{\prime}-F_{1}^{\prime}-w \cos \theta & =0 \\
M^{\prime}+F_{2} & =0
\end{aligned}
$$

where $F_{1}(s)$ and $F_{2}(s)$ are the axial and transverse components of the internal force $\mathbf{F}(s)$. This general beam model indeed contains both previously mentioned limit cases, as can be seen by degenerating the above equilibrium equations. On the one hand, if both the shear force $F_{2}$ and the bending moment $M$ are set equal to zero, a cable model is obtained

$$
\begin{aligned}
F_{1} \theta^{\prime}-w \sin \theta & =0 \\
F_{1}^{\prime}+w \cos \theta & =0
\end{aligned}
$$

which implies conservation of the horizontal component of the internal force: $F_{1} \sin \theta=$ Constant. On the other hand, if the curvature is assumed to be small, the classical linear beam model with uncoupled shear and axial forces is obtained

$$
\begin{aligned}
F_{2}^{\prime}-w \sin \theta & =0 \\
-F_{1}^{\prime}-w \cos \theta & =0 \\
M^{\prime}+F_{2} & =0 .
\end{aligned}
$$

Elimination of $F_{1}$ from (2) and (3) provides an expression for $F_{2}$, which, substituted into (4) and then (1), yields the beam equation

$$
E I\left(\theta^{\prime \prime \prime \prime} \theta^{\prime}-\theta^{\prime \prime} \theta^{\prime \prime \prime}+\theta^{\prime 3} \theta^{\prime \prime}\right)=w\left(-2 \theta^{\prime 2} \cos \theta+\theta^{\prime \prime} \sin \theta\right) .
$$

In the fundamental problem, this fourth order differential equation has to be solved on $s \in[0 ; \ell]$ with the following four boundary and constraining conditions

$$
\begin{aligned}
& \theta(0)=\theta_{p} \quad ; \quad \theta(\ell)=\theta_{q} \\
& \int_{0}^{\ell} \cos \theta d s=\Delta_{x} \quad ; \quad \int_{0}^{\ell} \sin \theta d s=\Delta_{y} .
\end{aligned}
$$


Because of the heavy analytical developments inherent to the application of the multiple scales method, the consideration of (7) is not convenient. Instead, a twice integrated version of this fourth order equation is used in further developments. Integration of adequate combinations of (2) and (3) yields

$$
\begin{aligned}
& F_{1}=H \sin \theta+(V-w s) \cos \theta \\
& F_{2}=H \cos \theta-(V-w s) \sin \theta
\end{aligned}
$$

where $H$ and $V$ are two integration constants, corresponding to the vertical and horizontal components of the internal force at $s=0$. Alternatively, these results could have been obtained from the consideration of the global equilibrium of the beam, with a Ritter-cut (2-b). The introduction of (11) into (4) and the consideration of (1) provide the classical second order non-linear beam equation

$$
E I \theta^{\prime \prime}=(V-w s) \sin \theta-H \cos \theta .
$$

In the fundamental problem, this equation has to be solved with (8) as boundary conditions, and $H$ and $V$ have to be determined in order to satisfy (9). The scope of the fundamental problem goes therefore beyond the one of the simpler problem with given values of $H$ and $V$.

\section{Dimensionless Formulation}

The dimensionless formulation of (12) is written as

$$
\varepsilon^{2} \vartheta^{\prime \prime}=(v-\xi) \sin \vartheta-h \cos \vartheta
$$

where $\xi=s / \ell$ is the dimensionless curvilinear abscissa and

$$
\begin{aligned}
\varepsilon^{2} & =\frac{E I}{w \ell^{3}} \\
\vartheta(\xi) & =\theta(\xi \ell) .
\end{aligned}
$$

Equation (13) indicates the existence of boundary layers, as the highest derivative of $\vartheta$ is multi- 
plied by a small parameter (Hinch, 1991; Kevorkian and Cole, 1996). The singularity of this class of problems is evident as the order of the differential equation (13) is decreased by 2 for $\varepsilon=0$.

All the force quantities are naturally scaled by the weight of the beam, the only intrinsic parameter with the dimension of a force in the considered problem

$$
\begin{gathered}
v=\frac{V}{w \ell} \quad ; \quad h=\frac{H}{w \ell} \\
f_{1}(\xi)=\frac{F_{1}(\xi \ell)}{w \ell} ; f_{2}(\xi)=\frac{F_{2}(\xi \ell)}{w \ell} \\
f(\xi)=\sqrt{f_{1}^{2}+f_{2}^{2}}=\sqrt{h^{2}+(v-\xi)^{2}} .
\end{gathered}
$$

Study of the fundamental problem consists in solving (13) for $\xi \in[0 ; 1]$, with the following boundary conditions

$$
\vartheta(0)=\theta_{p} \quad ; \quad \vartheta(1)=\theta_{q}
$$

and finding the values of $v$ and $h$ such that

$$
\int_{0}^{1} \cos \vartheta d \xi=\delta_{x} \quad ; \quad \int_{0}^{1} \sin \vartheta d \xi=\delta_{y}
$$

with $\delta_{x}=\Delta_{x} / \ell$, and $\delta_{y}=\Delta_{y} / \ell$. The solution of this problem is not unique (Schmeiser and Weiss, 1986), as solutions with curling of the beam could satisfy the same set of equations. We restrict consideration, however, to the solution without curl, which is the only one of interest in the context of the problem under investigation. Thus the axial force is restricted to be positive

$$
f_{1}=h \sin \vartheta+(v-\xi) \cos \vartheta \geq 0 .
$$

Also, for the sake of simplicity in the following developments, but without any loss of generality, it is assumed that

$$
\vartheta(\xi) \in[0 ; \pi]
$$




\section{Multiple Scales Solution}

Matching asymptotics methods consist in matching the inner solution inside the boundary layer and the outer solution corresponding to the unperturbed problem. Then, a composite solution, valid throughout the whole domain $\xi \in[0,1]$, can eventually be constructed (Hinch, 1991). On the contrary, multiple scales methods treat both scales simultaneously and provide at once a solution valid throughout the whole domain. Although it is in principle not necessary to appeal to the concept of inner and outer solutions, it simplifies the exposition of the method. Therefore, we first present the outer solution, then the multiple scales solution.

\section{Outer Solution}

The outer solution is obtained by constructing an asymptotic expansion for $\vartheta(\xi)$ of the form

$$
\vartheta(\xi)=h_{0}(\xi)+\varepsilon h_{1}(\xi)+\varepsilon^{2} h_{2}(\xi)+\ldots
$$

and substituting it into (13). Balancing the coefficients of the lowest power of $\varepsilon$ gives the leading order term of the outer solution

$$
h_{0}=\operatorname{arccot}^{+}\left(\frac{v-\xi}{h}\right)
$$

where $\operatorname{arccot}^{+}$is defined as

$$
\begin{array}{rlr}
\operatorname{arccot}^{+} x & =\operatorname{arccot} x & \text { for } x \geq 0 \\
=\pi+\operatorname{arccot} x & \text { for } x<0
\end{array}
$$

in order to satisfy (20) and to work with a continuous function. As expected, (22) corresponds to the catenary solution of a cable without bending stiffness (Irvine, 1975). However, we prefer to write the outer solution as

$$
h_{0}=\operatorname{arccot}^{+}\left((1-\xi) \cot \phi_{p}+\xi \cot \phi_{q}\right)
$$


where the integration constants $h$ and $v$ have been replaced by the temporary variables $\phi_{p}$ and $\phi_{q}$, representing the end inclinations of the cable in the catenary solution. In a non-trivial solution, $\phi_{p}$ and $\phi_{q}$ are necessarily different and are expressed as a function of $h$ and $v$ as

$$
v=\frac{\cot \phi_{p}}{\cot \phi_{p}-\cot \phi_{q}} \quad ; \quad h=\frac{1}{\cot \phi_{p}-\cot \phi_{q}} .
$$

As $\phi_{p}$ and $\phi_{q}$ are not necessarily equal to $\theta_{p}$ and $\theta_{q},(24)$ does not satisfy the boundary conditions. It is valid in the outer domain only, far from the beam ends. We expect, as confirmed later, that there exist two boundary layers, located in the vicinity of the beam ends, and that their extent is related to $\left|\phi_{p}-\theta_{p}\right|$ and $\left|\phi_{q}-\theta_{q}\right|$.

\section{Multiple Scales Approach}

Because there are two boundary layers, the multiple scales method should therefore consider three different scales: the physical scale $\xi$ and two stretched coordinates to represent each boundary layer. To consider these three scales simultaneously would result in a solution for which the interaction between both boundary layers is accounted for. It is desired to develop here a formulation giving a better accuracy for moderate values of $\varepsilon$, but not so large as to simultaneously involve both boundary layers. Such developments would anyway be rather tedious. We will therefore focus on each boundary layer separately, starting with the boundary layer close to $\xi=0$, and thus consider two scales: the slow varying coordinate $\xi$ and the fast varying coordinate

$$
\zeta=\xi / \varepsilon
$$

We seek a two-scale expansion for $\vartheta(\xi)$ of the form

$$
\vartheta(\xi, \zeta)=g_{0}(\xi, \zeta)+\varepsilon g_{1}(\xi, \zeta)+\varepsilon^{2} g_{2}(\xi, \zeta)+\ldots
$$

where $\zeta \in\left[0 ; \varepsilon^{-1}\right]$. As it is commonly assumed in the application of multiple scales methods (Kevorkian and Cole, 1996; Hinch, 1991), this series is supposed to be asymptotic for $\zeta=o(1)$. The total differentiation in the governing equation (13) is replaced by partial differentiations, obtained 
by means of the chain rule

$$
\frac{d^{2}}{d \xi^{2}}=\frac{\partial^{2}}{\partial \xi^{2}}+\frac{2}{\varepsilon} \frac{\partial^{2}}{\partial \xi \partial \zeta}+\frac{1}{\varepsilon^{2}} \frac{\partial^{2}}{\partial \zeta^{2}}
$$

Substitution into the governing equation and comparison of likewise powers of $\varepsilon$ yield partial differential equations for $g_{0}, g_{1}, \ldots$ :

$$
\begin{aligned}
\frac{\partial^{2} g_{0}}{\partial \zeta^{2}} & =(v-\xi) \sin g_{0}-h \cos g_{0} \\
\frac{\partial^{2} g_{1}}{\partial \zeta^{2}}+2 \frac{\partial^{2} g_{0}}{\partial \xi \partial \zeta} & =g_{1}(v-\xi) \cos g_{0}+g_{1} h \sin g_{0} .
\end{aligned}
$$

These equations can be solved recursively, starting from the lowest order in $\varepsilon$.

The following developments aim at the establishment of the leading order solution $g_{0}(\xi, \zeta)$. Integration of (28), previously multiplied by $\frac{\partial g_{0}}{\partial \zeta}$ gives

$$
\frac{1}{2}\left(\frac{\partial g_{0}}{\partial \zeta}\right)^{2}+(v-\xi) \cos g_{0}+h \sin g_{0}=A(\xi)
$$

where $A(\xi)$ is an integration function. Because of the restriction (19) on the sign of the axial force, $A(\xi)$ has to be positive on $[0 ; 1]$. It is not easy to solve (30) for any function $A(\xi)$. Nevertheless, its analytical expression can be derived from knowledge about the outer solution. Indeed there exists a value of $\varepsilon$ under which a fixed coordinate $\xi \neq 0$ lies in the outer domain. Thus, as $\varepsilon$ tends towards 0 , the inner solution has to degenerate into the outer one

$$
\lim _{\varepsilon \rightarrow 0} g_{0}(\xi, \zeta)=h_{0}(\xi)
$$

This justification is not valid in all applications of the multiple scales method, but it can be invoked here because it is applied in the context of a boundary layer problem. Differentiation of (31) with respect to $\zeta$ gives

$$
\frac{\partial}{\partial \zeta}\left[\lim _{\varepsilon \rightarrow 0} g_{0}(\xi, \zeta)\right]=\frac{\partial h_{0}}{\partial \zeta}
$$

The inversion of both mathematical operators is legitimate since the limits exist in both cases, giving 
therefore

$$
\lim _{\varepsilon \rightarrow 0} \frac{\partial g_{0}}{\partial \zeta}(\xi, \zeta)=0
$$

The limit for $\varepsilon$ tending towards 0 , applied to both sides of (30), simplifies to

$$
A(\xi)=(v-\xi) \cos h_{0}+h \sin h_{0}=\sqrt{(v-\xi)^{2}+h^{2}}=f(\xi)
$$

which shows that $A(\xi)$ actually corresponds to the resultant of the internal forces. After some simplifications (30) can be written as

$$
\frac{1}{2}\left(\frac{\partial g_{0}}{\partial \zeta}\right)^{2}=2 f \sin ^{2}\left(\frac{h_{0}-g_{0}}{2}\right)
$$

which can then be integrated to yield

$$
\ln \left(\tan \frac{h_{0}-g_{0}}{4}\right)=\ln (\tan B) \pm \zeta \sqrt{f}
$$

where $B(\xi)$ is an integration function. The negative sign has to be chosen in the \pm symbol, in order to provide a bounded solution for $\zeta \rightarrow+\infty$. The expression of the leading order solution is therefore

$$
g_{0}(\xi, \zeta)=h_{0}(\xi)+4 \arctan [\tan B(\xi) \exp (-\zeta \sqrt{f(\xi)})]
$$

The boundary condition at $\xi=0$, i.e. $\zeta=0$, yields

$$
B(0)=\frac{\theta_{P}-\phi_{p}}{4} .
$$

One particularity of the multiple scales method is that the consideration of the problem at a given order (here the leading order) is not sufficient to fully determine the solution. In this example the function $B(\xi)$ is just known by its value at the origin, and only consideration of the next order enables its determination. 


\section{Determination of $B(\xi)$}

The solution $g_{1}$ at the next order, which requires solving (29), depends on $g_{0}$ and therefore on $B(\xi)$. The optimal determination of $B(\xi)$ relies on Lindstedt's method (Lindstedt, 1883; Poincaré, 1957), that consists in setting secular terms in higher orders equal to zero, in order to keep the asymptoticness of (27) for $\zeta=o(1)$. These terms should be obtained by substituting the general expression of $g_{0}$ into (29)

$$
\frac{\partial^{2} g_{1}}{\partial \zeta^{2}}-\frac{\left(\tan ^{4} B-6 \tan ^{2} B e^{2 \zeta \sqrt{f}}+e^{4 \zeta \sqrt{f}}\right) f}{\left(\tan ^{2} B+e^{2 \zeta \sqrt{f}}\right)^{2}} g_{1}=-2 \frac{\partial^{2} g_{0}}{\partial \xi \partial \zeta}
$$

where

$$
-2 \frac{\partial^{2} g_{0}}{\partial \xi \partial \zeta}=\frac{4 e^{\zeta \sqrt{f}}}{r_{2}^{2} \sqrt{f}} \quad\left[\left(2 f B^{\prime} \sec B-\zeta \tan B \sqrt{f} f^{\prime}\right) r_{1}+\tan B f^{\prime} r_{2}\right]
$$

with

$$
r_{1}(\xi, \zeta)=e^{2 \zeta \sqrt{f}}-\tan ^{2} B, \quad r_{2}(\xi, \zeta)=e^{2 \zeta \sqrt{f}}+\tan ^{2} B
$$

Because (40) cannot be written as a sum of functions of $\zeta$, secular terms cannot be identified and Lindstedt's method has to be adapted. Here, the construction of $B(\xi)$ hinges on viewing (39) as the equation of a (non-linear) spring-mass system with a negative initial tangent stiffness, and a forcing term. To impose the asymptoticness of (27) for $\zeta=o(1)$, and hence to ensure that the function $g_{1}$ is bounded, the forcing term should be set to zero. However, the vanishing of (40) cannot be strictly fulfilled for any set $(\zeta, \xi)$, as $B$ is a function of $\xi$ only. Instead, we satisfy this condition close to the beam end (small values of $\zeta$ ), as further away in the boundary layer any error on $B(\xi)$ would anyway be wiped out by the exponentially decreasing function in (37). For this reason, a Taylor series expansion of (40) is considered around $\zeta=0$

$$
-2 \frac{\partial^{2} g_{0}}{\partial \xi \partial \zeta}=\frac{2}{\sqrt{f}}\left[4 \cos (2 B) f B^{\prime}+\sin (2 B) f^{\prime}\right]+o(\zeta)
$$

and $B(\xi)$ is chosen in such a way to cancel the first term of the expansion. These considerations lead 
to the formulation of a first-order differential equation for $B$

$$
B^{\prime}=-\frac{f^{\prime}}{f} \frac{\tan (2 B)}{4} .
$$

which can be solved with the boundary condition (38) to finally yield

$$
B(\xi)=\frac{1}{2} \arcsin \left(\sqrt{\frac{f(0)}{f(\xi)}} \sin \frac{\theta_{p}-\phi_{p}}{2}\right) .
$$

\section{Solution with Both Boundary Layers}

Because the three length scales are not considered simultaneously, the above solution is not a formal application of the multiple scales method. Indeed, the solution (37) obtained so far, should be valid throughout the whole domain in a formal multiple scales approach; however it does not embrace the second boundary layer. To this purpose, a similar reasonning for the second boundary layer is adopted and a composite solution (in the sense of a matched asymptotics method) is written as

$$
\begin{aligned}
\vartheta(\xi)=h_{0}(\xi) & +4 \arctan \left[\tan \left[\frac{1}{2} \arcsin \left(\sqrt{\frac{f(0)}{f(\xi)}} \sin \frac{\theta_{p}-\phi_{p}}{2}\right)\right] \exp \left(-\frac{\xi}{\varepsilon} \sqrt{f(\xi)}\right)\right] \\
& +4 \arctan \left[\tan \left[\frac{1}{2} \arcsin \left(\sqrt{\frac{f(1)}{f(\xi)}} \sin \frac{\theta_{q}-\phi_{q}}{2}\right)\right] \exp \left(-\frac{1-\xi}{\varepsilon} \sqrt{f(\xi)}\right)\right]
\end{aligned}
$$

where $h_{0}(\xi)$ and $f(\xi)$ are the inclination in the catenary solution and the resultant internal force given by (22) and (16), respectively. As expected, the boundary layer disappears when the inclination obtained in the catenary solution, $\phi_{p}$ or $\phi_{q}$, is equal to the corresponding boundary condition, $\theta_{p}$ or $\theta_{q}$. 


\section{Comparison with the Matched Asymptotics Method}

The developments leading to the matching asymptotics solution of the problem follow a very similar approach. They are summarized in Appendix. The composite solution is

$$
\begin{aligned}
\vartheta(\xi)=h_{0}(\xi) & +4 \arctan \left[\tan \frac{\theta_{p}-\phi_{p}}{4} \exp \left(-\frac{\xi}{\varepsilon} \sqrt{f(0)}\right)\right] \\
& +4 \arctan \left[\tan \frac{\theta_{q}-\phi_{q}}{4} \exp \left(-\frac{1-\xi}{\varepsilon} \sqrt{f(1)}\right)\right]
\end{aligned}
$$

The difference between this solution and that obtained with the multiple scales method lies essentially in the way the internal forces are accounted for within the boundary layer. The discrepancy between both methods is expected to be the largest for a fast varying function $f(\xi)$ in the vicinity of the beam ends. As the derivatives of $f$ at both ends correspond to the inclinations of the end reactions on the horizontal

$$
f^{\prime}(0)=\frac{-v}{\sqrt{v^{2}+h^{2}}} \quad \text { and } \quad f^{\prime}(1)=\frac{-(v-1)}{\sqrt{(v-1)^{2}+h^{2}}},
$$

the discrepancy should be the largest for end reactions having a vertical tendency.

\section{Validation}

The accuracy of the multiple scales solution is assessed with the problem of Fig. 3, which depicts half of a cable that is clamped at both ends. By symmetry, it is here represented by a cable with imposed inclinations at both ends $\left(\theta_{p}=\theta_{q}=\frac{\pi}{2}\right)$, and being free to move horizontally and vertically at each support, respectively. Because of these boundary conditions, the left reaction itself has to carry the whole weight of the cable $(V=w l$ or $v=1)$. Furthermore, it is assumed that the horizontal force at the left anchor is known $(H=w l$ or $h=1)$. This problem is not formulated within the framework of the considered fundamental problem, since the relative locations of the beam ends are not imposed.

The catenary solution and the corresponding end inclinations are obtained from (22) and (25)

$$
h_{0}(\xi)=\operatorname{arccot}^{+}(1-\xi) \quad ; \quad \phi_{p}=\frac{\pi}{4} \quad ; \quad \phi_{q}=\frac{\pi}{2}
$$


which indicates that there is no boundary layer at the right end, as $\phi_{q}=\theta_{q}$. The multiple scales and matched asymptotic solutions obtained from (44) and (45) are represented in Fig. 4 for $\varepsilon=0.15$.

They are compared to a reference solution, numerically obtained with a shooting method. In this method, the two-point boundary value problem is transformed into an initial one, by giving a guess for $\vartheta^{\prime}(0)$. An efficient ordinary differential equation solver (RK4) with adaptive step, is used to compute the solution of this well-posed initial value problem, and to assess the boundary condition at the other end of the beam. This shooting method requires thus to find the value(s) of $\vartheta^{\prime}(0)$ for which $\vartheta(1)-\theta_{q}=0$. This function is represented in Fig. 5. In view of its shape, high-order non-linear solvers such as as Newton-Raphson are not guaranteed to converge; hence, a bisection method was used to obtain the numerical solution of Fig. 4. In this example, the solution is unique, but more generally the function could cross several times the horizontal axis, indicating therefore the existence of multiple solutions.

As expected, the deflection of the cable, shown in Fig. 4, exhibits similar shapes, with a slight difference between the matched asymptotics solution and the multiple scales solution. The latter one shows a very good agreement with the numerical solution and the catenary solution fitted on the numerical solution in the outer domain.

\section{Solution of the Fundamental Problem}

The solution of the fundamental problem requires the consideration of the restraining equations (18), which have been ignored so far. These equations have to be solved for $h$ and $v$, or equivalently for $\phi_{p}$ and $\phi_{q}$.

First, in the absence of boundary layers, the restraining conditions would simply be

$$
\begin{aligned}
\delta_{x}\left(\phi_{p}, \phi_{q}\right) & =\frac{\sin \phi_{q}-\sin \phi_{p}}{\sin \left(\phi_{q}-\phi_{p}\right)} \\
\delta_{y}\left(\phi_{p}, \phi_{q}\right) & =\frac{1}{\cot \phi_{p}-\cot \phi_{q}} \ln \left(\frac{\tan \phi_{q} / 2}{\tan \phi_{p} / 2}\right)
\end{aligned}
$$

which is obtained by setting $\vartheta=h_{0}$ in (18). This set of transcendental equations representing the catenary solution cannot be solved analytically. The level curves of $\delta_{x}\left(\phi_{p}, \phi_{q}\right)$ and $\delta_{y}\left(\phi_{p}, \phi_{q}\right)$ are 
represented in Fig. 6-a for $\phi_{p}$ and $\phi_{q}$ in $[0, \pi]$, and $\phi_{p} \leq \phi_{q}$ in order to satisfy (19). In these plots, the points lying close to the diagonal edge correspond to $\phi_{p} \simeq \phi_{q}$, i.e. to highly taut cables or to a trivial straight catenary in the limit case.

The solution of (48) is geometrically represented as the intersection of the level curves corresponding to given values of $\delta_{x}$ and $\delta_{y}$. As any two level curves selected in each graph present a unique intersection, the solution of (48) is therefore unique.

It is naturally hopeless to solve the restraining conditions for $\phi_{p}$ and $\phi_{q}$, when the bending stiffness of the cable is considered. Contrary to the catenary case, substitution of the multiple scales solution (44) into (18) does not lead to an explicit solution. However, the resulting expressions of $\delta_{x}\left(\phi_{p}, \phi_{q}\right)$ and $\delta_{y}\left(\phi_{p}, \phi_{q}\right)$ can be obtained numerically. Figures 6-b and 6-c represent some examples with $\theta_{p}=$ $\theta_{q}=\frac{\pi}{2}$. Evidently, the patterns converge to those of $6-\mathrm{a}$, as $\varepsilon$ tends towards zero. Although plots of contour levels of $\delta_{x}$ and $\delta_{y}$ computed for others values of $\theta_{p}$ and $\theta_{q}$ would not exhibit the same symmetry as those shown in Figs. 6-b and 6-c, they would nonetheless also converge to those of 6-a, as $\varepsilon$ tends towards zero. The greyed zones in these figures, close to the axes $\phi_{p}=0$ and $\phi_{q}=\pi$ correspond to loose cables for which the internal forces decrease so fast in the boundary layers that the argument of one of the arcsin functions is larger than unity for some $\xi$, leading therefore to complex solutions. In these zones, the real part of $\delta_{x}\left(\phi_{p}, \phi_{q}\right)$ and $\delta_{y}\left(\phi_{p}, \phi_{q}\right)$ is represented but should be considered with care. This illustrates the limitations of the model. Physically, for a loose cable corresponding to values of $\phi_{p}$ and $\phi_{q}$ in the problematic ranges, the boundary layers occupy a wide part of the domain and may even interact, which is clearly in contradiction with the assumptions of the considered two-scale model.

In a practical application, the system of restraining conditions has to be solved numerically. The regularity of $\delta_{x}\left(\phi_{p}, \phi_{q}\right)$ and $\delta_{y}\left(\phi_{p}, \phi_{q}\right)$ indicates that many numerical methods will converge to the solution within a couple of iterations. The similarity of the level curves with the analytical case $(\varepsilon=0)$ suggests that the solution of the catenary problem may actually be used as a good starting point for the numerical approach.

Figure 7 represents the solution of the cable with horizontal supports, obtained for $\varepsilon=0.10$, $\delta_{x}=0, \delta_{y}=0.97$. A Newton-Raphson solver converges (tolerance of $10^{-6}$ on the function estimate) to $\phi_{p}=0.810, \phi_{q}=2.331$ after two iterations and $0.07 \mathrm{~s}$ CPU running on an AMD Turion(tm) processor, 
Tech.ML-34, 1.79GHz, RAM 1.00Gb. The same procedure is applied with the matched asymptotics solution and yields $\phi_{p}=0.881, \phi_{q}=2.261$, with similar computation time.

The internal forces and displacements obtained with these semi-analytical methods are compared to results obtained by a full numerical analysis. Because we now have to cope with a non-linearity similar to that presented in Fig. 5, a high order numerical method does not systematically converge for this problem. Instead, the reference solution is obtained with a dedicated algorithm combining bisection and Newton-Raphson methods. It results in $\phi_{p}=0.797, \phi_{q}=2.345$ with $2.47 \mathrm{~s}$ CPU on the same processor; the internal forces and displacements resulting from this numerical computation are also plotted in Fig. 7.

The matched asymptotics and multiple scales methods give very close results for displacement based quantities, which is expected since the problem is kinematically controlled. However, when considering the internal forces, the matched asymptotics method leads to less precise estimates (up to $15 \%$ off, for the axial force). It is not surprising to observe a larger discrepancy on the axial force, since, unlike the shear force or bending moment, it is not proportional to a certain derivative of $\vartheta(\xi)$, but rather to ratios $\vartheta^{\prime \prime \prime}(\xi) / \vartheta^{\prime}(\xi)$ and $\sin \vartheta(\xi) / \vartheta^{\prime}(\xi)$.

\section{Conclusions}

We have applied the multiple scales method to the analysis of a beam with a small bending stiffness, and compared the results to those obtained with the matched asymptotics method, which is the commonly used perturbation technique to solve that problem. Because of the necessity to identify secular terms, or to develop any equivalent formulation as proposed in this paper, the multiple scales method requires the consideration of the second order solution, and consequently somewhat more involved analytical developments. However, the approximate solution exhibits a similar analytical form for both perturbation methods, and they require therefore a similar computation time. The multiple scales solution is shown to be a refined generalization of the matched asymptotic one, allowing a better representation of the internal forces within the boundary layer.

The proposed multiple scales solution might be seen as an inexpensive way to improve the quality of the solution of the considered problem, in particular for moderate values of the reduced beam 
stiffness $(\varepsilon \simeq 0.10)$ and when axial forces are concerned.

The analysis of this problem suggests that other classes of problems involving boundary layers, which have been only treated in the framework of the matched asymptotics method, might benefit from the application of a multiple scales approach.

\section{Acknowledgments}

The theory presented in this paper has been developed during an invited stay of V. Denoël at the Commonwealth Scientific and Industrial Research Organisation (CSIRO, Perth, Australia), under the agreement of the Belgian Fund for Scientific Research and the University of Liège (Belgium). These institutions are therefore warmly acknowledged. The authors would also like to recognize fruitful discussions with Dr Thomas Richard (CSIRO).

\section{Appendix}

The outer solution $h_{0}(\xi)$ presented in section is also valid for the matched asymptotic expansion. The inner solution results from the definition (26) of the same stretched coordinate as in the multiple scales approach. The major difference is that now, the differential equation is written with this stretched coordinate only

$$
\vartheta^{\prime \prime}(\zeta \varepsilon)=(v-\zeta \varepsilon) \sin \vartheta(\zeta \varepsilon)-h \cos \vartheta(\zeta \varepsilon)
$$

We seek an asymptotic matching of the form

$$
\vartheta(\zeta \varepsilon)=G_{0}(\zeta)+\varepsilon G_{1}(\zeta)+\ldots
$$

The comparison of the coefficients of the lowest powers of $\varepsilon$ yields

$$
G_{0}^{\prime \prime}(\zeta)=v \sin G_{0}(\zeta)-h \cos G_{0}(\zeta)
$$


Similarly to (30), a first integral is written

$$
\frac{1}{2} G_{0}^{\prime 2}(\zeta)+v \cos G_{0}(\zeta)+h \sin G_{0}(\zeta)=a
$$

where $a$ is now a simple constant. Again, the solution of this differential equation does not take a simple form for any value of $a$. For this reason, a matching between the inner and outer solutions is performed. According to Van Dyke's rule, the matching is imposed by setting

$$
\begin{aligned}
\lim _{\varepsilon \rightarrow 0} G_{0}(\zeta)=\lim _{\varepsilon \rightarrow 0} h_{0}(\zeta \varepsilon) & =\lim _{\varepsilon \rightarrow 0} \operatorname{arccot}^{+} \frac{v-\zeta \varepsilon}{h}=h_{0}(0) \\
\lim _{\varepsilon \rightarrow 0} G_{0}^{\prime}(\zeta)=\lim _{\varepsilon \rightarrow 0} \frac{d}{d \zeta} h_{0}(\zeta \varepsilon) & =\lim _{\varepsilon \rightarrow 0} \operatorname{arccot}^{+} \frac{\varepsilon h}{h^{2}+(v-\zeta \varepsilon)^{2}}=0
\end{aligned}
$$

The application of these limits to (52) yields $a=v \cos h_{0}(0)+h \sin h_{0}(0)=f(0)$, and (52) can be solved similarly to what was done previously. A constant of integration $b$, similar to $B(\xi)$, has to be introduced. Its value is determined uniquely from the boundary conditions, and is evidently found to be equal to $B(0)$. An analogous development for the second boundary layer, and the assembly of the outer and both inner solutions give finally the composite solution (45).

\section{References}

Ascher, U. and Weiss, R. (1983). "Collocation for singular pertubation problems 1: 1st-order systems with constant coefficients." Siam Journal On Numerical Analysis, 20(3), 537-557. Times Cited: 40.

Bieniasz, L. K. (2008). "Adaptive solution of bvps in singularly perturbed second-order odes, by the extended numerov method combined with an iterative local grid h-refinement." Applied Mathematics and Computation, 198(2), 665-682. Bieniasz, L. K.

Clark, J. D., Fraser, W. B., and Stump, D. M. (2001). "Modelling of tension in yarn package unwinding.” Journal Of Engineering Mathematics, 40(1), 59-75. Times Cited: 1.

Denoël, V. (2008). "Advantages of a semi-analytical approach for the analysis of an evolving 
structure with contacts." Communications in Numerical Methods in Engineering. in press, doi: 10.1002/cnm.1059.

Esipova, V. (1975). "The asymptotic behavior of solutions of the general boundary value problem for singularly perturbed systems of ordinary differential equations of conditionally stable type." Differential Equations, 11(11), 1457-1465.

Flaherty, J. E. and O’Malley, R. E. (1982). "Singularly-perturbed boundary-value-problems for nonlinear systems, including a challenging problem for a non-linear beam." Lecture Notes in Mathematics, 942, 170-191.

Hinch, E. J. (1991). Perturbation Methods, Vol. 1. Cambridge University Press, Cambridge.

Irvine, H. M. (1975). "Statics of suspended cables.” Journal of the Engineering Mechanics DivisionAsce, 101(3), 187-205. Times Cited: 11.

Irvine, M. (1993). “Local bending stresses in cables." International Journal of Offshore and Polar Engineering, 3(3), 172-175.

Jain, M. K., Iyengar, S. R. K., and Subramanyam, G. S. (1984). "Variable mesh methods for the numerical-solution of 2-point singular perturbation problems." Computer Methods in Applied Mechanics and Engineering, 42(3), 273-286. Times Cited: 25.

Kevorkian, J. and Cole, J. D. (1996). Multiple Scale and Singular Perturbation Methods.

Kumar, M., Singh, P., and Mishra, H. K. (2007). “A recent survey on computational techniques for solving singularly perturbed boundary value problems." International Journal of Computer Mathematics, 84(10), 1439-1463. Kumar, Manoj Singh, Pitam Mishra, Hradyesh Kumar.

Lindstedt, A. (1883). "Sur la forme des expressions des distances mutuelles dans le problème des trois corps." Comptes Rendus De L Academie Des Sciences.

Maier, M. R. (1986). "An adaptative shooting method for singularly perturbed boundary-valueproblems." Siam Journal on Scientific and Statistical Computing, 7(2), 418-440. Times Cited: 7. 
Plunkett, R. (1967). "Static bending stresses in catenaries and drill strings." Journal of Engineering for Industry, 89(1), 31-\&.

Poincaré, H. (1957). Les méthodes nouvelles de la mécanique céleste, Vol. II. Dover, New-York.

Rao, S. C. S. and Kumar, M. (2007). "B-spline collocation method for nonlinear singularly-perturbed two-point boundary-value problems." Journal of Optimization Theory and Applications, 134(1), 91-105. Rao, S. C. S. Kumar, M.

Rienstra, S. W. (1987). “Analytical approximations for offshore pipelaying problems.

Schmeiser, C. (1985). "Finite deformations of thin beams - asymptotic analysis by singular perturbation-methods." Ima Journal Of Applied Mathematics, 34(2), 155-164. Times Cited: 8.

Schmeiser, C. and Weiss, R. (1986). "Asymptotic analysis of singular singularly perturbed boundaryvalue-problems." Siam Journal on Mathematical Analysis, 17(3), 560-579. Times Cited: 17.

Stump, D. M. and Fraser, W. B. (2000). "Bending boundary layers in a moving strip." Nonlinear Dynamics, 21(1), 55-70. Times Cited: 2.

Stump, D. M. and van der Heijden, G. H. M. (2000). "Matched asymptotic expansions for bent and twisted rods: applications for cable and pipeline laying." Journal Of Engineering Mathematics, 38(1), 13-31. Times Cited: 2.

Stynes, M. and Oriordan, E. (1986). "A finite-element method for a singulary perturbed boundaryvalue problem." Numerische Mathematik, 50(1), 1-15. Times Cited: 25.

Timoshenko, S. P. and Goodier, J. N. (1987). Theory of Elasticity. McGraw-Hill Book Company, New-York, third edition edition.

Vasileva, A. B. (1973). Asymptotic expansions of solutions of singularly perturbed equations. Moscow.

Wolfe, P. (1991). "Asymptotic analysis of a rod with small bending stiffness." Quarterly of Applied Mathematics, 49(1), 53-65. Times Cited: 2. 
Wolfe, P. (1993). "Hanging cables with small bending stiffness." Nonlinear Analysis-Theory Methods \& Applications, 20(10), 1193-1204. Times Cited: 3. 